\title{
ACCESO A LAS PLATAFORMAS DE PRÉSTAMO DIGITAL: INTEGRACIÓN VS. ATOMIZACIÓN
}

\author{
Access to digital lending platforms: \\ Integration vs, atomization
}

Elena Sánchez-Muñoz

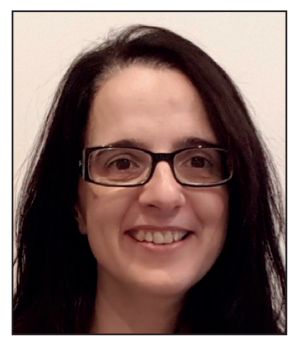

Elena Sánchez-Muñoz, licenciada en Documentación por la Universitat Oberta de Catalunya y en Filología Inglesa por la Universidad de Santiago de Compostela, es doctoranda en el programa Formación en la Sociedad del Conocimiento de la Universidad de Salamanca. Funcionaria del cuerpo Facultativo Superior de Bibliotecas de la Xunta de Galicia, desde 2014 trabaja en el Servizo do Sistema de Bibliotecas en la implantación y coordinación del catálogo colectivo de la Red de Bibliotecas Públicas de Galicia, así como en el proyecto Galiciale para el préstamo de ebooks.

http://orcid.org/0000-0001-8375-7911

Consellería de Cultura, Educación e Ordenación Universitaria Servizo do Sistema de Bibliotecas Cidade da Cultura. Monte Gaiás, s/n. 15707 Santiago de Compostela, España elena.sanchez.munoz@xunta.gal

\section{Resumen}

Las bibliotecas públicas españolas se enfrentan a la escasa implantación del préstamo digital entre sus lectores. La oferta de este servicio fragmentada en varias plataformas y su falta de integración con otros recursos como el catálogo online supone un mayor esfuerzo de adaptación para los usuarios y coloca a las propias bibliotecas en situación de desventaja. Por medio de un sistema de indicadores se evalúan las 3 principales plataformas de préstamo de libro electrónico españolas [OdiloTK (de Odilo), Xebook (de Xercode) e iBiblio (de Libranda)] a través de su implantación en eBiblio Galicia, Catálogo gallego de GaliciaLe (CGG) y eBookVigo/eBook DiCoruña, respectivamente. Se evidencia las diferencias existentes entre ellas y la necesidad de proporcionar un acceso único e integrado con las funciones que aportan los softwares de descubrimiento y las APIs.

\section{Palabras clave}

Préstamo digital; Libros electrónicos, Plataformas de préstamo de libro electrónico; Programas de descubrimiento; Software de descubrimiento; Bibliotecas públicas; Opacs; Servicios de descubrimiento; Usuarios; Lectores; Herramientas de descubrimiento; Indicadores.

\section{Abstract}

Spanish public libraries are faced with the scarce implementation of digital loans among their readers. The offer of this service, fragmented in several platforms, and its lack of integration with other resources such as the online catalog implies a greater effort of adaptation for users and places the libraries themselves at a disadvantage. Through a system of indicators, the 3 main Spanish e-book loan platforms [OdiloTK (from Odilo), Xebook (from Xercode), and iBiblio (from Libranda)] are evaluated through their implementation in eBiblio Galicia, Galician Catalog of GaliciaLe (CGG) and eBookVigo / eBook DiCoruña, respectively. There is evidence of the differences between them and a need to provide a unique and integrated access to the functions provided by discovery tools and APIs.

\section{Keywords}

eLending; Ebooks; Ebook lending platforms; Discovery tools; Discovery layers; Discovery software; Public libraries; Opacs; Web-scale discovery services, Patrons; Readers; Indicators.

Sánchez-Muñoz, Elena (2018). "Acceso a las plataformas de préstamo digital: integración vs. atomización". El profesional de la información, v. 27, n. 3, pp. 582-594. 


\section{Introducción}

Los datos relativos al libro electrónico en España publicados en los informes eBiblio 2015', eBiblio 2016 y eBiblio2017² (MECD, 2016a, 2017a, 2018) muestran una baja implantación del préstamo digital en las bibliotecas públicas españolas.

Si comparamos los 51.720 .491 préstamos de materiales tradicionales según Bibliotecas públicas españolas en cifras 2015 (MECD, 2016b) con los 246.115 de ebooks en ese año, el porcentaje de estos últimos sobre el total asciende al $0,47 \%$. A pesar del incremento en un $43,6 \%$ del préstamo digital en 2016 (con 353.439 préstamos) las cifras todavía están muy lejos de las alcanzadas en Estados Unidos, donde el porcentaje de préstamos de libros electrónicos en las bibliotecas públicas supuso un $8 \%$ del total durante 2016, cuadruplicando las cifras de 2011 (Hoffert, 2017).

Uno de los factores que explican estos bajos índices en España es la limitada oferta de títulos en versión electrónica. Según el informe Panorámica de la edición española de libros 2016 (MECD, 2017b) el número de ISBNs inscritos en 2016 fue de 86.000 . El 70,65\% corresponde a libros en soporte papel y el $29,35 \%$ a ebooks. En un análisis pormenorizado, el pdf representa el 36,8\% de la edición electrónica, el ePub el $28,3 \%$, otros formatos como scorm o html el $24,7 \%$, mobi/ azv el 3,9\% y el cd-rom, dvd o cd-i el 6,3\%. Es decir, de los 25.241 libros que se publican en digital, sólo 16.431 tienen un formato con cabida en las plataformas de préstamo digital ( $p d f$ o ePub) de las bibliotecas públicas españolas frente a los 60.759 en papel. Quedan fuera de éstas los libros en formato azv de Kindle, por lo que muchos usuarios con dispositivos de Amazon no pueden descargarse las obras que ofertan estas plataformas. A esta situación estructural, se suma la renuencia de una parte del sector editorial a dar acceso a algunos títulos, especialmente novedades y más vendidos, para el préstamo en bibliotecas (Alonso-Arévalo; Cordón-García, 2015).

Un segundo factor son las propias plataformas de préstamo digital. Por un lado, en éstas confluyen algunas de las dificultades técnicas a las que se enfrentan los lectores, especialmente las relacionadas con la descarga de libros: enlazado y desenlazado de dispositivos, obtención de un ID, instalación de Adobe digital editions y de apps, autorización de equipos, etc. Según un estudio realizado en las bibliotecas de Derbyshire (Reino Unido), el $27 \%$ de los usuarios afirma que los problemas técnicos les han hecho abandonar el préstamo de un ebook en alguna ocasión y un $5 \%$ manifiesta que esto le sucede a menudo (Martindale; Willett; Jones, 2015).

Por otro lado, la atomización del acceso implica que los lectores deben aprender a manejarse en varios entornos (el del propio servicio de préstamo digital, el del opac y el de otros recursos electrónicos). En los casos más extremos, varias de las plataformas con mayor implantación en España (OdiloTK de Odilo, Xebook de Xercode e iBiblio de Libranda) llegan a ofertarse en una misma biblioteca pública en accesos separados en lo que constituye una balcanización de entornos (Kelley, 2012) y una experiencia de consulta fragmentada que obliga a buscar los contenidos a través de varias interfaces con sus propias especificidades (Alonso-Arévalo; Cordón-García, 2015).
A lo largo del presente artículo compararemos las funciones de las interfaces, la experiencia de préstamo y de lectura en las tres principales plataformas de préstamo digital españolas a través de un sistema de indicadores que aplicaremos a los cuatro entornos ofertados por las bibliotecas públicas gallegas:

- eBiblio Galicia: eBiblio es el entorno gestionado con OdiIoTK e impulsado por el Ministerio de Educación, Cultura y Deporte (MECD) en colaboración con las comunidades autónomas. En Galicia, eBiblio Galicia se presenta en el marco del proyecto GaliciaLe.

- Catálogo gallego de GaliciaLe: impulsado por la Xunta de Galicia, se integra igualmente en el proyecto GaliciaLe y se sustenta en la tecnología Xebook (basada en Koha).

- eBookVigo (Ayuntamiento de Vigo) y eBook DiCoruña (Deputación Provincial da Coruña), ambas gestionadas con iBiblio.

El objetivo de esta comparativa es poner de relieve las diferencias existentes entre estos entornos y la consiguiente curva de aprendizaje a la que se enfrentan los lectores.

Esta atomización, unida a las dificultades técnicas inherentes al préstamo digital, son elementos que pueden condicionar el éxito de estos servicios y explicar, en parte, los bajos índices de préstamo de libro electrónico en España.

Finalmente, expondremos las soluciones tecnológicas que permiten dar un acceso integrado y que ya se están aplicando en las bibliotecas públicas estadounidenses.

\section{Metodología}

Con el objetivo de evaluar los servicios, información y funciones de las plataformas de préstamo digital, hemos creado un sistema de 55 indicadores agrupados bajo 12 epígrafes (tabla 0).

Para los indicadores dedicados al análisis de la interfaz (epígrafes 1-10) hemos tomado como referencia los modelos de evaluación de softwares de descubrimiento y catálogos online elaborados por Muñoz-Egido y Hernández-Pérez (2016); Novotny (2004); Deodato (2015); Chickering y Yang (2014), y Yang y Wagner (2010).

Así mismo, los indicadores dedicados a la circulación y la lectura (epígrafes 11 y 12) se basan en los propuestos por Codina (2008) dentro de los parámetros de ergonomía y usabilidad (facilidad, flexibilidad, visión de estatus y convenciones, a los que hemos añadido adaptabilidad en el caso de la lectura).

El análisis de la interfaz concentra un total de 66 puntos, en tanto que la circulación y lectura aglutinan los 34 puntos restantes (tabla 0).

Para los indicadores de los epígrafes 1, 2, 4-7, 11, 12 hemos asignado 0 en caso de no cumplimiento, 1 en caso de cumplimiento y 2 si la plataforma presenta información, servicios, o funciones añadidas. Los indicadores de los epígrafes 3, 8-10 tienen naturaleza binaria (0 si la plataforma no cumple el indicador, 1 si lo cumple).

Con el fin de evaluar cuantitativamente las diferencias y similitudes existentes entre las plataformas de préstamo, hemos aplicado este sistema de indicadores a las versiones actualmente implantadas en las bibliotecas públicas gallegas 
y a las funciones que ofrece OdiloTK para eBiblio Galicia, Xebook para el Catálogo gallego de Galiciale (en adelante, CGG) e iBiblio para eBookVigo y eBook DiCoruña.

\section{Análisis}

\subsection{Navegación y recuperación}

La aplicación de los indicadores de navegación muestra una clara diferencia entre la puntuación de eBiblio Galicia y CGG por un lado y, eBookVigo/eBook DiCoruña, por otro. El análisis pormenorizado de cada indicador, sin embargo, refleja la disparidad también existente entre los dos primeros entornos.

Hemos valorado positivamente la incorporación de funciones como la búsqueda simple accesible en todas las páginas, la ordenación por relevancia y la presencia de búsqueda fuzzy y stemming. Todas ellas proporcionan una experiencia similar a la de los entornos en internet a los que ya está acostumbrado el lector y contribuyen a cumplir sus expectativas, producto de la confluencia de su experiencia de búsqueda en motores como Google y plataformas de comercio electrónico como Amazon (Bawden; Vilar, 2006) o, en los términos acuñados por Dempsey (2005), “Amazoogle" y "Googlezon".

eBiblio Galicia y CGG presentan búsqueda simple y avanzada, en tanto que eBookVigo/eBook DiCoruña sólo la simple. Ésta está disponible en todas las páginas de eBiblio Galicia y de eBookVigo/eBook DiCoruña. En el caso de CGG, sin embargo, está ausente en la visualización de cada registro bi- bliográfico obligando a retroceder a la página anterior para hacer una nueva búsqueda.

Las diferencias también son perceptibles en las opciones de truncamiento, operadores booleanos y ayudas al usuario, todas ellas ausentes en eBookVigo/eBook DiCoruña. eBiblio Galicia admite el uso de comodines y presenta la búsqueda avanzada estructurada en combos con los que el lector puede elegir entre dos operadores, pero sin ninguna orientación sobre su uso. CGG admite el empleo de comodines y operadores booleanos y posibilita búsquedas por rango y proximidad, todas ellas acompañadas de ayudas.

\begin{tabular}{|c|c|c|c|}
\hline & \\
\hline & eBiblio Galicia & CGG & $\begin{array}{l}\text { eBookVigo/ } \\
\text { eBook DiCoruña }\end{array}$ \\
\hline $\begin{array}{l}\text { 1.1. Búsqueda simple y avanzada } \\
(+) \text { : búsqueda simple accesible en todas las páginas }\end{array}$ & 2 & 1 & 1 \\
\hline $\begin{array}{l}\text { 1.2. Truncamiento y operadores booleanos } \\
(+) \text { : ayudas al usuario }\end{array}$ & 1 & 2 & 0 \\
\hline $\begin{array}{l}\text { 1.3. Funciones para la mejora de las búsquedas: fuzzy y stemming } \\
(+) \text { : inclusión de frases orientativas }\end{array}$ & 0 & 2 & 0 \\
\hline $\begin{array}{l}\text { 1.4. Lenguaje documental } \\
\text { (+): similitud con los lenguajes naturales }\end{array}$ & 2 & 2 & 2 \\
\hline $\begin{array}{l}\text { 1.5. Navegación a través de un menú de categorías temáticas } \\
\text { (+): estructuración de las categorías en varios niveles }\end{array}$ & 2 & 1 & 2 \\
\hline $\begin{array}{l}\text { 1.6. Ordenación de resultados por fecha, autor y título } \\
\text { (+): ordenación por relevancia }\end{array}$ & 2 & 2 & 2 \\
\hline $\begin{array}{l}\text { 1.7. Filtrado de resultados por autor, materia, editorial, año de publicación e idioma } \\
(+) \text { : filtrado por disponibilidad y formato }\end{array}$ & 2 & 1 & 0 \\
\hline $\begin{array}{l}\text { 1.8. Navegación semántica entre registros de un mismo autor y/o materia } \\
(+) \text { : presencia de opciones añadidas de navegación entre registros }\end{array}$ & 1 & 2 & 1 \\
\hline Total & $12 / 16$ & $13 / 16$ & $8 / 16$ \\
\hline
\end{tabular}

$0=$ No; 1=Sí; $2=$ Valor añadido (+) 
Las funciones fuzzy y stemming tan sólo están presentes en CGG. Incorpora, además, una frase orientativa cuando ningún registro contiene el término exacto introducido por el usuario (figura 1).

La mayor similitud entre entornos la hallamos en el uso de lenguajes naturales controlados para clasificar los fondos. eBiblio Galicia utiliza descriptores propios y CGG adopta la Clasificación Decimal Universal (CDU) (habitualmente empleada en las bibliotecas públicas españolas para la organización de sus colecciones) si bien la notación se traduce al lenguaje natural de forma transparente para el usuario. eBookVigo/ eBook DiCoruña emplean IBIC (International Book Industry Categories), un sistema de clasificación de materias con orientación comercial basado en el lenguaje natural.

Los cuatro entornos ordenan los resultados por relevancia, fecha, autor o título, pero eBiblio Galicia proporciona más posibilidades de filtrado: al formato y a los campos tradicionales de descripción bibliográfica con los que también cuenta CGG, eBiblio Galicia suma el refinamiento por palabras y por disponibilidad.

Finalmente, eBiblio Galicia posibilita la navegación entre registros de un mismo autor o materia, a lo que CGG añade la navegación por editorial y serie. En eBookVigo/eBook DiCoruña se limita a registros de un mismo autor y, adicionalmente, por editorial y lengua.

Tabla 2. Descripción

\begin{tabular}{|l|c|c|c|}
\cline { 2 - 3 } \multicolumn{1}{c|}{} & $\begin{array}{c}\text { eBiblio } \\
\text { Galicia }\end{array}$ & CGG & $\begin{array}{c}\text { eBookVi- } \\
\text { go/eBook } \\
\text { DiCoruña }\end{array}$ \\
\hline $\begin{array}{l}\text { Descripción bibliográfica básica } \\
\text { (+): enriquecimiento mediante fuentes externas }\end{array}$ & $2 / 2$ & $1 / 2$ & $1 / 2$ \\
\hline
\end{tabular}

0=No; 1=Sí; 2=Valor añadido (+)

Tabla 3. Exportación

\begin{tabular}{|l|c|c|c|}
\cline { 2 - 4 } \multicolumn{1}{c|}{} & $\begin{array}{c}\text { eBiblio } \\
\text { Galicia }\end{array}$ & CGG & $\begin{array}{c}\text { eBookVigo/ } \\
\text { eBook } \\
\text { DiCoruña }\end{array}$ \\
\hline 3.1. URL del registro & 1 & 1 & 1 \\
\hline 3.2. Cita de un recurso en varios formatos & 0 & 1 & 0 \\
\hline 3.3. Integración con gestores de referencias & 0 & 1 & 0 \\
\hline 3.4. Envío de registros por correo & 0 & 1 & 0 \\
\hline 3.5. Envío de búsquedas por correo & 1 & 1 & 0 \\
\hline 3.6. Sindicación de contenidos & 0 & 1 & 0 \\
\hline Total & $2 / 6$ & $6 / 6$ & $1 / 6$ \\
\hline
\end{tabular}

\subsection{Descripción de contenidos}

Los cuatro entornos incorporan resúmenes, materias y los campos tradicionales de descripción bibliográfica, pero sólo eBiblio Galicia enriquece los registros con enlaces a entradas de la Wikipedia para los autores.

\subsection{Exportación de información}

En este epígrafe encontramos una gran disparidad en las puntuaciones, motivada posiblemente por el hecho de que Xebook tiene una mayor implantación en las bibliotecas universitarias, cuyos usuarios utilizan los gestores de referencias y citas de forma más habitual.

Los cuatro entornos proporcionan un URL permanente que facilita la exportación y difusión de cada registro y lo identifica unívocamente por medio de una ID. En CGG y en eBookVigo/eBook DiCoruña, ésta se visualiza en la barra de direcciones. En eBiblio Galicia, sin embargo, es necesario generar el enlace pulsando un botón (figura 2).

De los cuatro entornos, sólo CGG genera citas APA y MLA y está integrado con gestores de referencias que posibilitan la exportación de los registros a Refworks, Endnote y Mendeley.

\subsection{Información sobre disponibilidad, formatos y opciones de lectura}

La inclusión de esta información, sobre todo la relativa a la disponibilidad, resulta especialmente relevante para el usuario, por lo que el acceso inmediato y detallado a la misma añade valor a la plataforma.

Los cuatro entornos cumplen los indicadores básicos, pero sólo eBiblio Galicia alcanza la puntuación máxima gracias a la incorporación de información añadida (tabla 4). 


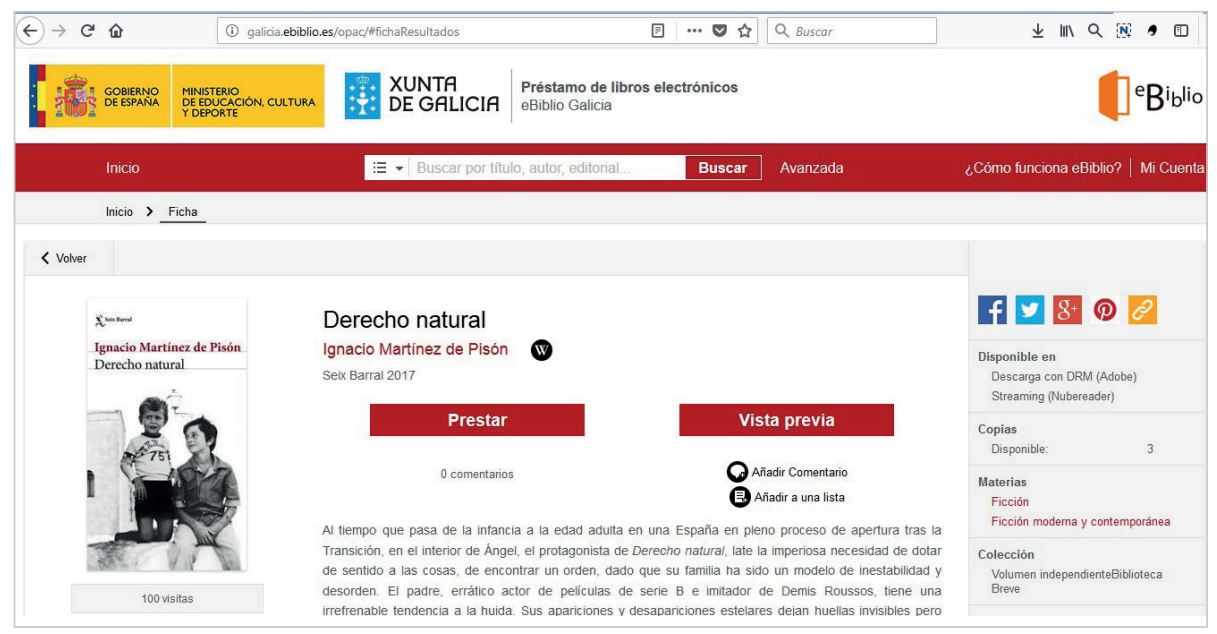

Figura 2. Ejemplo de registro de eBiblio Galicia. El URL del registro no se genera en la barra de direcciones sino mediante el icono naranja situado en la parte central derecha de la pantalla. al que va a adquirir. En su adaptación a las plataformas de préstamo, hemos valorado si éstas presentan información que sirva para despertar el interés del lector por otros títulos e incrementar la sensación de seguridad y confianza en el servicio (libros leídos por otros usuarios, títulos más prestados, etc.). eBiblio Galicia emplea varios elementos de cross selling: número de visitas a cada registro, "Otros usuarios también vieron", "Otros usuarios también leyeron" y, como valor añadido, personalización de las sugerencias con "Recomendados para ti". CGG incorpora un carrusel de títulos más prestados,

\subsection{Estrategias de marketing}

El marketing online está presente en buena parte de las plataformas de comercio electrónico: previsualización de una obra, exhibición de novedades y recomendaciones en sliders, posicionamiento web, libros similares a los que ha adquirido el cliente o personalización de sugerencias son algunos ejemplos. En las plataformas de préstamo, su utilización puede contribuir a una mayor difusión y rotación de la colección y proporciona al usuario una experiencia similar a la del comercio online.

La técnica de cross selling tiene por objeto hacer que el cliente compre un segundo producto similar o complementario muestra obras similares al registro consultado por el lector y "Otros usuarios también leyeron", pero, al igual que eBookVigo/eBook DiCoruña, no personaliza las sugerencias. Estos dos últimos entornos emplean el cross selling para mostrar obras devueltas recientemente por otros usuarios.

Otra técnica, la exhibición de libros en sliders, se utiliza de manera desigual. Tanto CGG como eBiblio Galicia muestran títulos seleccionados manualmente por el administrador, si bien en el caso de eBiblio Galicia, el sistema no genera automáticamente el carrusel para las obras más prestadas que sí muestra CGG. eBookVigo/eBook DiCoruña dan cabida a dos espacios, ambos para mostrar obras seleccionadas manualmente.

Tabla 4. Información sobre disponibilidad, formatos y opciones de lectura

\begin{tabular}{|c|c|c|c|}
\hline & \\
\hline & $\begin{array}{l}\text { eBiblio } \\
\text { Galicia }\end{array}$ & CGG & $\begin{array}{l}\text { eBookVigo/eBook } \\
\text { DiCoruña }\end{array}$ \\
\hline $\begin{array}{l}\text { 4.1. Información sobre disponibilidad } \\
\text { (+): información relativa a tiempos de espera en caso de reserva }\end{array}$ & 2 & 1 & 1 \\
\hline $\begin{array}{l}\text { 4.2. Información sobre formatos } \\
\text { (+): presencia de esta información en la lista de resultados }\end{array}$ & 2 & 2 & 1 \\
\hline $\begin{array}{l}\text { 4.3. Información sobre opciones de lectura } \\
\text { (+): presencia de esta información en la lista de resultados }\end{array}$ & 2 & 1 & 1 \\
\hline Total & $6 / 6$ & $4 / 6$ & $3 / 6$ \\
\hline
\end{tabular}

$0=$ No; 1=Sí; 2=Información añadida (+)

Tabla 5. Estrategias de marketing

\begin{tabular}{|c|c|c|c|}
\hline & $\begin{array}{l}\text { eBiblio } \\
\text { Galicia }\end{array}$ & CGG & $\begin{array}{l}\text { eBookVigo/eBook } \\
\text { DiCoruña }\end{array}$ \\
\hline $\begin{array}{l}\text { 5.1. Empleo de técnicas de cross selling } \\
\text { (+): personalización de las sugerencias }\end{array}$ & 2 & 1 & 1 \\
\hline $\begin{array}{l}\text { 5.2. Presencia de sliders y/o espacios en la página principal para mostrar una selección de títulos } \\
\text { (+): Variedad y número de carruseles y/o espacios (más de } 2 \text {, automatizados y manuales) }\end{array}$ & 1 & 2 & 1 \\
\hline $\begin{array}{l}\text { 5.3. Vista previa de los contenidos } \\
\text { (+): previsualización sin identificación del usuario }\end{array}$ & 2 & 0 & 1 \\
\hline $\begin{array}{l}\text { 5.4. Acciones de mejora de posicionamiento web } \\
\text { (+): posicionamiento entre los } 50 \text { primeros puestos en los principales buscadores }\end{array}$ & 1 & 0 & 0 \\
\hline Total & $6 / 8$ & $3 / 8$ & $3 / 8$ \\
\hline
\end{tabular}

$0=$ No; $1=S i ́ ; 2=$ Valor añadido (+) 
La previsualización de los contenidos brinda al lector una experiencia similar al "hojeo" en una librería o biblioteca. La posibilidad de acceder a esta función sin identificación previa y desde la lista de resultados (sin necesidad de hacer dos clics para llegar a las páginas de muestra) contribuye a atraer a nuevos usuarios hacia los contenidos de la plataforma.

eBiblio Galicia cumple estos indicadores en su totalidad y eBookVigo/eBook DiCoruña lo hacen parcialmente al dar acceso a las primeras páginas desde el registro individual de cada obra previa identificación del lector. CGG tiene un comportamiento similar al de eBookVigo/eBook DiCoruña con la limitación de proporcionar la previsualización sólo para los libros en formato pdf.

El empleo de estrategias de marketing online en las plataformas de préstamo digital contribuye a la difusión de la colección y proporciona al lector un entorno similar al del comercio electrónico

Para el posicionamiento web, hemos considerado las acciones de optimización llevadas a cabo en eBiblio Galicia, en CGG y en eBookVigo/eBook DiCoruña, que tienen por objeto mejorar su visibilidad y ocupar una posición destacada en los buscadores. Así, en lo que respecta a las etiquetas de metadatos <title>, <description> y <keywords> del código fuente, eBiblio Galicia hace un mejor uso de éstas:

- <title> contiene el nombre de la Comunidad Autónoma;

- <description> da una visión global "Plataforma para la gestión y el préstamo de contenido digital (ebooks, audio y vídeo)";

- <keywords> abarca un amplio número de términos (préstamo libros electrónicos, ebook, streaming, Bibliotecas, ebiblio, etc.).

CGG incorpora únicamente <title>, si bien la información que contiene no es identificativa del recurso. eBookVigo/ eBook DiCoruña consignan las tres etiquetas, pero dejan vacías < description $>$ y <keywords $>$.

Con el fin de obtener el número de backlinks, hemos empleado Open Link Profiler con bajos resultados en los cuatro entornos: 15 páginas apuntan a eBiblio Galicia y sólo 2 a CGG, a eBookVigo y eBook DiCoruña respectivamente.

Para el posicionamiento entre los 50 primeros puestos hemos introducido los términos "préstamo libro electrónico" en los cinco principales buscadores (Google, Bing, Yahoo, Ask.com y $A O L$ ), pero ninguna de las plataformas alcanza esta posición.

\subsection{Diseño responsivo}

Los cuatro entornos superan el test de optimización para móviles de Google aunque sólo eBookVigo/eBook DiCoruña lo hacen sin errores. Para eBiblio Galicia se informa de incidencias puntuales en la carga debido a fallos de redireccionamiento y en $C G G$ no se pudieron cargar varios recursos a causa de los problemas detectados en las imágenes.

Para la revisión de las media queries hemos empleado CSS Validator del World Wide Web Consortium (W3C) con un resultado positivo en todos los casos.

En el análisis del atributo viewport hemos comprobado su construcción en el código fuente conforme a la sintaxis recomendada por W3C: <meta name="viewport" content="width=device-width, initial-scale $=1.0^{\prime \prime}>$.

En la visualización en dispositivos móviles hemos valorado el uso de menús desplegables, la ausencia o minimización del scroll y la no superposición de imágenes.

\subsection{Accesibilidad}

Para analizar el nivel de accesibilidad de los cuatro entornos, hemos aplicado el test TAW (test de accesibilidad web). Éste mide el grado de accesibilidad de un sitio web en relación con las Web content accessibility guidelines (WCAG) 2.0 (W3C, 2008) de la siguiente forma:

- WCAG 2.0 A: cumple todos los puntos de verificación de prioridad 1 (no existen problemas automáticos de nivel 1 y se descartan todos los problemas manuales de esta prioridad).

- WCAG 2.0 AA: cumple todos los puntos de verificación de prioridad 1 y 2 (no existen problemas automáticos de prioridad 1 y 2 y se descartan los problemas manuales indicados en estas prioridades).

- WCAG 2.0 AAA: cumple todos los puntos de verificación de prioridad 1, 2 y 3 (no existen problemas automáticos ni manuales de ninguna prioridad).

La aplicación del test TAW a las páginas de inicio de cada uno de los cuatro entornos da como resultado la existencia de errores automáticos de prioridad 1 y 2 , así como ma-

Tabla 7. Accesibilidad

\begin{tabular}{|l|c|c|c|}
\cline { 2 - 4 } & $\begin{array}{c}\text { eBiblio } \\
\text { Galicia }\end{array}$ & CGG & $\begin{array}{c}\text { eBookVigo/eBook } \\
\text { DiCoruña }\end{array}$ \\
\hline Superación del test TAW & $0 / 2$ & $0 / 2$ & $0 / 2$ \\
\hline
\end{tabular}

$0=$ No; $1=S i$, pero con errores (nivel A, AA); 2=Sí, sin errores (AAA) 


\begin{tabular}{|c|c|c|c|}
\hline & $\begin{array}{l}\text { eBiblio } \\
\text { Galicia }\end{array}$ & CGG & $\begin{array}{c}\text { eBookVigo/eBook } \\
\text { DiCoruña }\end{array}$ \\
\hline 8.1. Acceso por número o ID de usuario & 1 & 1 & 0 \\
\hline 8.2. Visualización de los datos de usuario & 0 & 1 & 0 \\
\hline 8.3. Modificación de los datos personales por el usuario & 0 & 0 & 1 \\
\hline 8.4. Información relativa a las transacciones de circulación & 1 & 1 & 1 \\
\hline 8.5. Historial de préstamos & 1 & 1 & 0 \\
\hline 8.6. Historial de previsualizaciones & 0 & 0 & 1 \\
\hline 8.7. Historial de búsquedas & 1 & 1 & 0 \\
\hline 8.8. Visualización de notificaciones relativas a novedades, paradas en el servicio, etc. & 1 & 0 & 0 \\
\hline 8.9. Visualización de los dispositivos vinculados & 0 & 0 & 1 \\
\hline 8.10. Enlazado/desenlazado de dispositivos desde la sesión web & 0 & 0 & 1 \\
\hline 8.11. Lista de favoritos & 1 & 1 & 0 \\
\hline Total & $6 / 11$ & $6 / 11$ & $5 / 11$ \\
\hline
\end{tabular}

$0=$ No; $1=$ Sí

nuales, por lo que ninguna de ellas alcanza el nivel mínimo (WCAG $2.0 \mathrm{~A}$ ).

\subsection{Sesión personal del usuario}

En este epígrafe hemos evaluado los servicios que presenta la sesión personal del lector.

Hemos valorado positivamente el login por medio del número de carné de biblioteca. La identificación a través de la dirección de correo electrónico se ha evaluado negativamente porque obliga a que cada usuario tenga una cuenta diferenciada y no repetible y dificulta el acceso de lectores infantiles sin una dirección o que utilizan la de sus progenitores. No obstante, debemos señalar que en la comunicación entre eBiblio y el SIGB (sistema integrado de gestión de bibliotecas) de las bibliotecas a través de un webservice, éste está configurado de modo que si el usuario no tiene una dirección de correo electrónico asociada en el directorio LDAP $^{3}$ (Lightweight directory access protocol) no puede acceder a eBiblio.

Dentro de la sesión personal de eBiblio Galicia y de CGG, el lector puede visualizar los avisos remitidos por el administrador. No obstante, CGG sólo permite el envío de notificaciones individualizadas a cada usuario consultables dentro de su sesión, en tanto que eBiblio Galicia posibilita la remisión de avisos tanto de forma individualizada como masiva (útil para la recepción de información sobre novedades, paradas en el servicio, etc.), visibles en la sesión personal del usuario y desde su correo electrónico en caso de que el administrador también haya escogido esta opción de envío.

En lo que respecta al historial de búsquedas, eBiblio Galicia y CGG tienen un comportamiento más homogéneo. La consulta temporal de las búsquedas recientes se conserva en eBiblio Galicia durante la sesión personal y en CGG 24 horas. El lector también puede almacenarlas permanentemente para próximas sesiones o eliminarlas.

Tan sólo eBookVigo/eBook DiCoruña proporcionan información relativa a los dispositivos vinculados a la cuenta del lector y posibilitan el enlazado y desenlazado de los mismos desde la sesión web. De este modo, si el usuario se ha desprendido de su móvil o tablet sin haberlo desenlazado antes, puede hacerlo posteriormente (figura 3 ).

\subsection{Participación del usuario}

Los lectores de los cuatro entornos pueden compartir información sobre un libro a través de las redes sociales. Como función

Tabla 9. Participación

\begin{tabular}{|l|c|c|c|}
\cline { 2 - 4 } \multicolumn{1}{c|}{} & $\begin{array}{c}\text { eBiblio } \\
\text { Galicia }\end{array}$ & CGG & $\begin{array}{c}\text { eBookVigo/eBook } \\
\text { DiCoruña }\end{array}$ \\
\hline 9.1. Redes sociales & 1 & 1 & 1 \\
\hline 9.2. Etiquetado social & 0 & 1 & 0 \\
\hline 9.3. Puntuación & 1 & 1 & 1 \\
\hline 9.4. Comentarios & 1 & 1 & 0 \\
\hline 9.5. Listas públicas & 1 & 0 & 0 \\
\hline Total & $4 / 5$ & $4 / 5$ & $2 / 5$ \\
\hline
\end{tabular}

$0=$ No; 1 =Sí

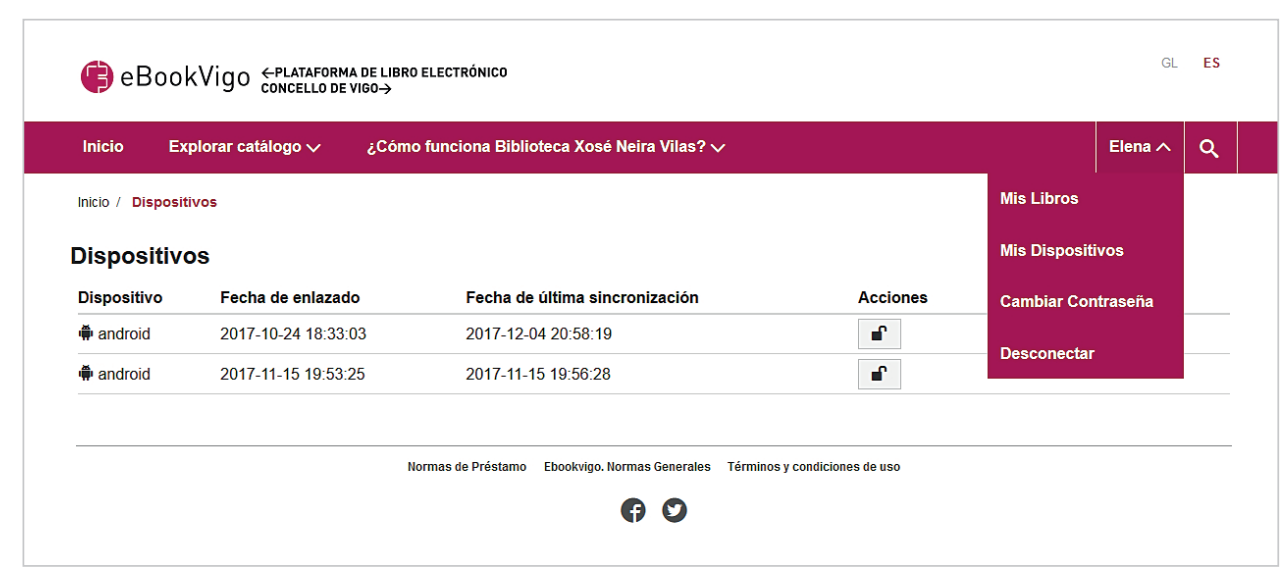

Figura 3. Visualización en la sesión web de eBookVigo de los dispositivos enlazados a la cuenta del lector 
Tabla 10. Apps

\begin{tabular}{|l|c|c|}
\cline { 2 - 3 } \multicolumn{1}{c|}{} & eBiblio Galicia & $\begin{array}{c}\text { eBookVigo/eBook } \\
\text { DiCoruña }\end{array}$ \\
\hline 10.1. Instalación sin acceso a contenidos del dispositivo & 0 & - \\
\hline 10.2. Valoración de los usuarios 3=< & 1 & - \\
\hline 10.3. Navegación transparente por el catálogo & 1 & - \\
\hline 10.4. Mismas funciones o superiores a las de sesión web y visor en streaming & 1 & - \\
\hline Total & $3 / 4$ & - \\
\hline
\end{tabular}

$0=$ No; $1=$ Sí

añadida, el visor de eBiblio Galicia permite compartir durante la lectura en streaming una palabra o fragmento del contenido.

La participación del usuario se articula de manera similar en eBiblio Galicia y CGG: éste puede publicar comentarios (previa moderación del administrador) y puntuar una obra, si bien sólo CGG permite la asignación de etiquetas o tags y únicamente eBiblio Galicia posibilita la publicación de listas por parte del lector.

\subsection{Apps}

Bajo este epígrafe hemos valorado la app de eBiblio (versión 2.0.12 en Android y 1.6.3 en iOS) y las apps de eBookVigo y de eBook DiCoruña (versión 2.5.0 en Android y 2.4.0 en iOS). CGG no dispone de app, hecho que hemos considerado una limitación que puede suplirse, en parte, con la app de $A D E$ (Adobe digital editions), Bluefire o Aldiko, pero que hace que el lector pierda la vinculación con la plataforma.

Para la valoración de las apps por sus usuarios, hemos asignado 0 si es menor de 3 y 1 si es mayor o igual a 3 . La de eBiblio obtiene una puntuación de 3,4 sobre 5 en Google Play y de 2,1 sobre 5 en App Store (con una media ponderada de 3,35). La puntuación otorgada por los usuarios en Android para eBookVigo y eBook DiCoruña es de 4,4 si bien iOS informa de que ambas apps no han recibido suficientes valoraciones para mostrar una media.

Para la navegación en el catálogo desde la app, hemos evaluado que sea transparente para el lector y que éste no deba abandonar el entorno de la propia app para su exploración.

Las apps de los tres entornos (eBiblio Galicia y eBookVigo/ eBook DiCoruña) brindan las mismas funciones que la sesión web del usuario y el visor de lectura en streaming, llegando incluso a superarlas en algunos aspectos: la de eBiblio Galicia incorpora la búsqueda de términos en un diccionario y, a diferencia de la sesión web, permite devolver los libros descargados. Así mismo, las apps de eBookVigo/eBook DiCoruña añaden opciones de personalización de las que carece el visor en streaming (marcadores, notas, ajuste del brillo, orientación de la pantalla y selección del color de fondo).

\subsection{Circulación}

El análisis de las acciones asociadas al préstamo, reserva y devolución se ha basado en cuatro indicadores propuestos por Codina (2008) dentro de los parámetros de ergonomía y usabilidad. Cada uno de ellos se ha valorado con 0 en caso de no cumplimiento, 1 en caso de cumplimento parcial y 2 si se cumple en la totalidad de los procesos.
Para el indicador "Facilidad" hemos valorado que las acciones relacionadas con todas las transacciones de circulación sean accesibles y fáciles de llevar a cabo.

Con "Flexibilidad" hemos evaluado la posibilidad de realizar estas acciones de diversas formas. eBiblio Galicia posee una mayor flexibilidad porque no sólo permite prestar y reservar desde el registro individual del libro sino también desde la lista de resultados evitando al lector un segundo clic. Así mismo, ofrece más opciones para autorizar un equipo en $A D E$ ya que el usuario puede seleccionar como proveedor Adobe ID (previa obtención de unas credenciales de Adobe) o, de manera más sencilla, Odilo y emplear las mismas claves de acceso que en eBiblio Galicia.

Las cuatro plataformas posibilitan la devolución anticipada desde el entorno web para la lectura en streaming. En caso de descarga del libro, eBiblio Galicia es la mejor posicionada ya que permite la devolución desde $A D E$ o desde la app, a diferencia de $C G G$, que sólo ofrece la posibilidad de hacerlo desde $A D E$, y de eBookVigo/eBook DiCoruña, que no proporcionan ninguna de estas opciones.

Con el indicador "Visión de estatus" hemos valorado la vi-

Tabla 11. Circulación

\begin{tabular}{|c|c|c|c|}
\hline & $\begin{array}{l}\text { eBiblio } \\
\text { Galicia }\end{array}$ & CGG & $\begin{array}{c}\text { eBookVigo/ } \\
\text { eBook DiCoruña }\end{array}$ \\
\hline \multicolumn{4}{|c|}{ 11.1. Facilidad } \\
\hline Préstamo & 2 & 2 & 2 \\
\hline Reserva & 2 & 2 & 2 \\
\hline Devolución & 2 & 2 & 2 \\
\hline \multicolumn{4}{|c|}{ 11.2. Flexibilidad } \\
\hline Préstamo & 2 & 0 & 0 \\
\hline Reserva & 2 & 0 & 0 \\
\hline Devolución & 2 & 1 & 0 \\
\hline \multicolumn{4}{|c|}{ 11.3. Visión de estatus } \\
\hline Préstamo & 2 & 1 & 0 \\
\hline Reserva & 2 & 0 & 1 \\
\hline Devolución & 2 & 2 & 2 \\
\hline \multicolumn{4}{|c|}{ 11.4. Convenciones } \\
\hline Préstamo & 1 & 0 & 2 \\
\hline Reserva & 2 & 2 & 2 \\
\hline Devolución & 1 & 0 & 1 \\
\hline Total & $22 / 24$ & $12 / 24$ & $14 / 24$ \\
\hline
\end{tabular}

$0=$ No; $1=S i ́$, parcialmente; $2=S i ́$, en su totalidad 
sión no ambigua de la situación en los procesos de circulación, especialmente las indicaciones del estado de las acciones que está llevando a cabo el usuario. Los cuatro entornos informan al lector acerca del tiempo que resta para la finalización del período de préstamo $y$, una vez agotado, la devolución se produce automáticamente sin necesidad de que el usuario realice ninguna acción. eBiblio Galicia, sin embargo, es la que presenta una mejor visión de estatus para el conjunto de las transacciones: la lista de resultados indica al lector si tiene en préstamo o ha reservado alguna de las obras recuperadas en una búsqueda y el botón "Prestar" es sustituido por el literal "Reservar" cuando un título no está disponible (figura 4). Así mismo, proporciona información sobre el tiempo aproximado de espera en el registro individual del libro.

Esta visión de estatus se ve dificultada en eBookVigo/eBook DiCoruña porque, a pesar de incorporar el botón "Bajo reserva" junto a los títulos no disponibles, éste redirige al registro individual desde donde hay que pulsar, de nuevo, el botón "Reservar".

Para el indicador "Convenciones" hemos valorado los iconos y mensajes que aparecen durante las transacciones de circulación. Observamos una mayor homogeneidad y un mejor uso de las convenciones en eBiblio Galicia y en eBookVigo/ eBook DiCoruña. En ambas, el botón de préstamo se sitúa próximo a la imagen de la cubierta. Por el contrario, en CGG se ubica lejos de ésta. Esta disposición puede llevar al usuario que quiere tomar un libro en préstamo a pinchar sobre los iconos informativos en lugar de hacerlo sobre el botón de préstamo (figura 5). A esta dificultad se añade la ausencia de ayudas contextuales.
eBookVigo/eBook DiCoruña emplean más eficazmente los mensajes ya que cada una de las opciones de lectura se acompaña de un enlace a un breve texto explicativo. Así mismo, al pulsar el botón "Descargar", el sistema genera un aviso que indica el tamaño del archivo e informa al usuario de que puede leer el libro en streaming o desde la app y de que tras la descarga no podrá utilizar la opción de devolución anticipada.

Para la reserva y devolución, los cuatro entornos utilizan iconos y mensajes claros, pero es en la devolución anticipada previa descarga en donde estas convenciones se usan de manera desigual. Tanto en eBiblio Galicia como en CGG el icono de devolución desaparece, si bien eBiblio Galicia incorpora una breve explicación y un enlace a una página en la que se muestran los pasos para hacer efectiva la devolución anticipada. En eBookVigo/eBook DiCoruña el botón de devolución permanece inactivo proporcionando una información contextual más clara que, sin embargo, se acompaña de un mensaje cuyo contenido es confuso ya que no hace alusión a que la causa por la que el usuario no puede devolverlo es que lo ha descargado previamente (figura 6).

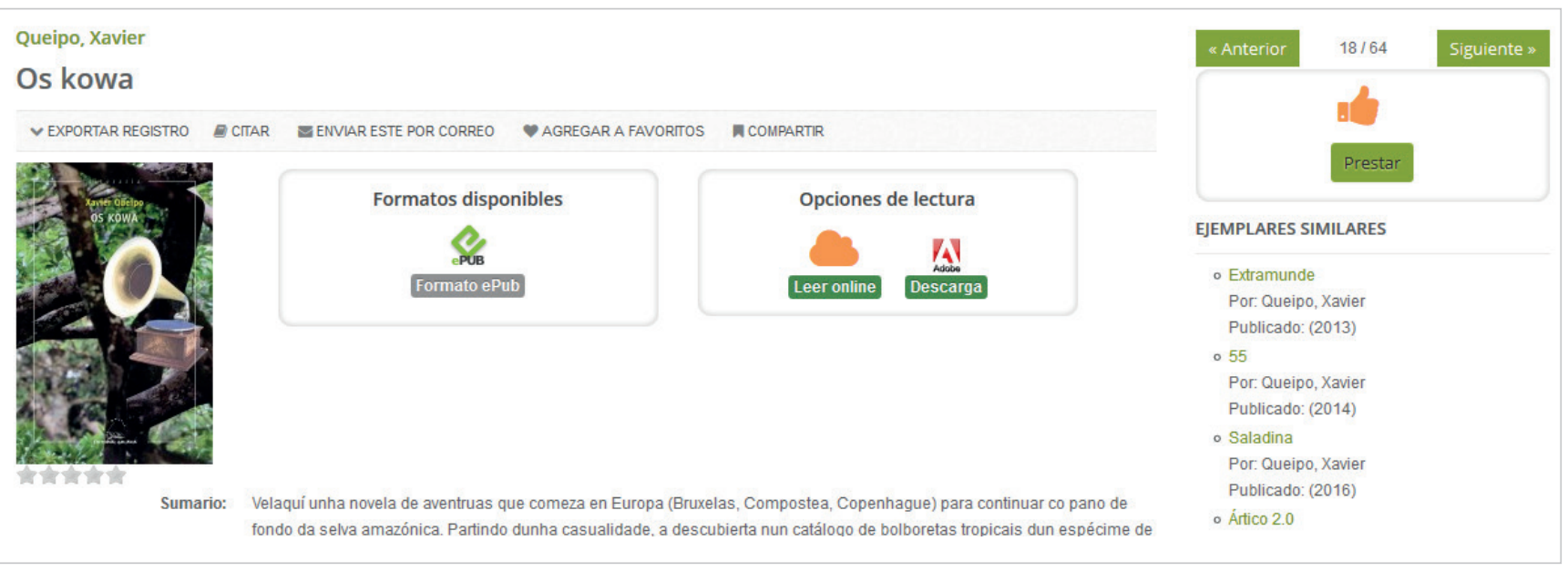

Figura 5. Ubicación del botón de préstamo en CGG en la parte superior derecha de la pantalla 


\section{Mis Libros Muestras Reservas}

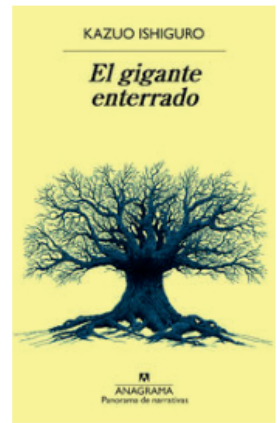

$\unrhd$ El gigante enterrado

Kazuo Ishiguro

Días restantes de préstamo: 6

Este formato de libro no permite realizar devoluciones. El libro desaparecerá de tu biblioteca una vez agotado el periodo de préstamo.

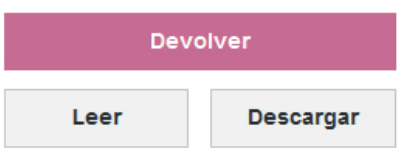

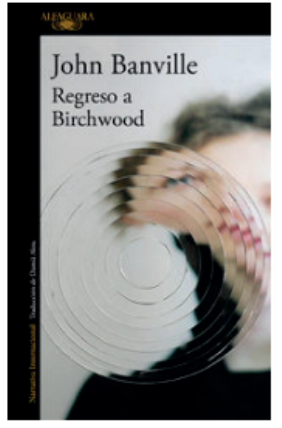

Regreso a Birchwood

John Banville

Días restantes de préstamo: 20 Este formato de libro no permite realizar devoluciones. El libro desaparecerá de tu biblioteca una vez agotado el periodo de préstamo.

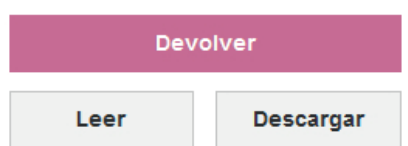

Figura 6. Visualización en eBookVigo del botón “Devolver” tras la descarga del ebook y mensaje advirtiendo de que la devolución no está permitida

\subsection{Lectura}

Para el análisis de las acciones asociadas a la lectura hemos tomado como referencia cinco indicadores propuestos por Codina (2008) dentro de los parámetros de ergonomía y usabilidad. Cada uno de éstos se ha valorado con 0 en caso de no cumplimiento, 1 en caso de cumplimento parcial y 2 si se cumple en la totalidad del proceso de lectura.

Con el indicador "Facilidad" hemos valorado que las acciones relacionadas con la lectura, tanto en dispositivos móviles como en PC, sean accesibles y fáciles de llevar a cabo.

Para el indicador "Flexibilidad" hemos evaluado las opciones de lectura, así como la sincronización en varios dispositivos. Los cuatro entornos permiten la lectura en streaming y mediante descarga. Los usuarios de eBiblio Galicia y de eBookVigo/eBook DiCoruña tienen la posibilidad de leer en sus dispositivos sin necesidad de conexión a internet gracias a las apps de las que disponen estas tres plataformas. CGG no proporciona este servicio, por lo que el lector debe descargarse, como alternativa, apps para la lectura de libros con DRM (Aldiko, Bluefire o la app de $A D E$ ). En lo que respecta a la sincronización, sólo eBiblio Galicia y eBookVigo/ eBook DiCoruña permiten enlazar dispositivos a la cuenta del usuario de modo que el lector puede iniciar la lectura en cualquiera de ellos y retomarla en el mismo punto donde la dejó y con los mismos subrayados, notas y marcadores.

Para la "Visión de estatus" hemos evaluado positivamente la visión no ambigua de las acciones relacionadas con la lectu-

Tabla 12. Lectura

\begin{tabular}{|l|c|c|c|}
\cline { 2 - 4 } \multicolumn{1}{c|}{} & $\begin{array}{c}\text { eBiblio } \\
\text { Galicia }\end{array}$ & CGG & $\begin{array}{c}\text { eBookVigo/ } \\
\text { eBook DiCoruña }\end{array}$ \\
\hline 12.1. Facilidad & 2 & 2 & 2 \\
\hline 12.2. Flexibilidad & 2 & 1 & 2 \\
\hline 12.3. Visión de estatus & 2 & 1 & 2 \\
\hline 12.4. Convenciones & 1 & 1 & 2 \\
\hline 12.5. Adaptabilidad & 2 & 1 & 1 \\
\hline Total & $9 / 10$ & $6 / 10$ & $9 / 10$ \\
\hline
\end{tabular}

$0=$ No; 1 =Sí, parcialmente; $2=$ Sí, en su totalidad ra obteniendo un resultado desigual. Tanto la app de eBiblio Galicia como el visor para streaming disponen de una barra de progreso, marcadores de posición y datos estadísticos como el tiempo dedicado a la lectura o el número de páginas leídas por hora. El visor de eBookVigo/eBook DiCoruña, al igual que la app, muestra una barra de progreso, pero no cuenta con los marcadores que presenta la app. El visor de CGG incorpora marcadores, pero no una barra de progreso.

Con el indicador "Convenciones" hemos valorado la utilización de iconos convencionales y la presencia de mensajes de ayuda contextual. En estos últimos hay una menor uniformidad ya que sólo el visor de eBookVigo/eBook DiCoruña y el de CGG muestran textos emergentes asociados a cada icono, si bien en CGG aparecen en inglés.

\section{El resultado de la aplicación a los cua- tro entornos del sistema de indicadores muestra cuantitativamente la falta de homogeneidad a la que debe hacer fren- te el lector}

En "Adaptabilidad" hemos evaluado la capacidad de adaptación a las necesidades del usuario durante la lectura. eBiblio Galicia muestra mayores posibilidades de personalización ya que tanto la app como el visor de streaming permiten cambiar la configuración (fuente, tamaño, espaciado, brillo y color de fondo), anotar, subrayar, incorporar marcadores y buscar un término dentro del contenido del libro. La app añade la posibilidad de seleccionar una palabra para obtener su definición en un diccionario o su entrada en la Wikipedia. Por contra, el visor de eBookVigo/eBook DiCoruña presenta opciones reducidas frente a la app, en tanto que CGG no incorpora la función de búsqueda.

\section{Resultados y discusión}

De la aplicación del sistema de indicadores se desprende la escasa uniformidad de las funciones y servicios que proporciona cada uno de los cuatro entornos. La tabla 13 resume las puntuaciones. 


\begin{tabular}{|c|c|c|c|}
\hline & $\begin{array}{c}\text { eBiblio Galicia } \\
\text { (OdiloTK de Odilo) }\end{array}$ & $\begin{array}{c}\text { CGG } \\
\text { (Xebook, de Xercode) }\end{array}$ & $\begin{array}{c}\text { eBookVigo/eBook } \\
\text { DiCoruña } \\
\text { (iBiblio de Libranda) }\end{array}$ \\
\hline 1. Navegación y recuperación & $12 / 16$ & $13 / 16$ & $8 / 16$ \\
\hline 2. Descripción de contenidos & $2 / 2$ & $1 / 2$ & $1 / 2$ \\
\hline 3. Exportación de información & $2 / 6$ & $6 / 6$ & $1 / 6$ \\
\hline 4. Información sobre disponibilidad, formatos y opciones de lectura & $6 / 6$ & $4 / 6$ & $3 / 6$ \\
\hline 5. Estrategias de marketing & $6 / 8$ & $3 / 8$ & $3 / 8$ \\
\hline 6. Diseño responsivo & $5 / 6$ & $3 / 6$ & $5 / 6$ \\
\hline 7. Accesibilidad & $0 / 2$ & $0 / 2$ & $0 / 2$ \\
\hline 8. Sesión personal del usuario & $6 / 11$ & $6 / 11$ & $5 / 11$ \\
\hline 9. Participación del usuario & $4 / 5$ & $4 / 5$ & $2 / 5$ \\
\hline 10. Apps & $3 / 4$ & - & $3 / 4$ \\
\hline Subtotal indicadores valoración interfaces & $46 / 66$ & $40 / 66$ & $31 / 66$ \\
\hline 11. Circulación & $22 / 24$ & $12 / 24$ & $14 / 24$ \\
\hline 12. Lectura & $9 / 10$ & $6 / 10$ & $9 / 10$ \\
\hline Subtotal indicadores circulación y lectura & $31 / 34$ & $18 / 34$ & $23 / 34$ \\
\hline Total & $77 / 100$ & $58 / 100$ & $54 / 100$ \\
\hline
\end{tabular}

A pesar de que CGG y eBookVigo/eBook DiCoruña obtienen prácticamente la misma puntuación global, existen claras diferencias entre los resultados que alcanzan los cuatro entornos en cada epígrafe.

De este modo, las puntuaciones parciales en los indicadores para la valoración de las interfaces (46 puntos eBiblio Galicia, 40 CGG y 31 eBookVigo/eBook DiCoruña) reflejan esta disparidad, con una mayor dispersión en los epígrafes 1,3 y 5 .

En un análisis más pormenorizado, eBiblio Galicia logra un mejor resultado debido al valor añadido que ofrece (más funciones, servicios e información y menos errores), destacando especialmente en los indicadores de información sobre disponibilidad, formatos y opciones de lectura (epígrafe 4) y estrategias de marketing (epígrafe 5). CGG supera a los demás entornos en exportación de la información (epígrafe 3).

En lo que respecta a la navegación y recuperación (epígrafe 1) y participación del usuario (epígrafe 9) eBiblio Galicia y CGG obtienen una valoración similar, superior a la de eBookVigo/eBook DiCoruña. Sin embargo, en diseño responsivo (epígrafe 6) y apps (epígrafe 10) eBiblio Galicia y eBookVigo/ eBook DiCoruña tienen un comportamiento más homogéneo, principalmente, porque las tres disponen de app.

La mayor uniformidad entre estos entornos la encontramos en los indicadores de descripción (epígrafe 2) y sesión personal del usuario (epígrafe 8).

La aplicación de los indicadores de circulación (epígrafe 11) vuelve a mostrar una disimilitud considerable entre eBiblio Galicia, por un lado, y CGG y eBookVigo/eBook DiCoruña por otro (22, 12 y 14 puntos respectivamente). En el caso de la lectura (epígrafe 12), los resultados son más homogéneos entre eBiblio Galicia y eBookVigo/eBook DiCoruña (9 puntos) frente a CGG (6).

Esta evidente disparidad no supondría un obstáculo dada la diversidad de recursos disponibles en internet a la que muchos usuarios están acostumbrados. El mayor problema radica en la oferta de un mismo servicio (el préstamo digital) desde un mismo tipo de bibliotecas (públicas), pero desde entornos muy distintos entre sí que requieren la identificación del lector en cada uno de ellos para prestar, reservar o devolver un libro. La inversión de tiempo y esfuerzo para superar la curva de aprendizaje y la resolución de incidencias de naturaleza diversa en cada plataforma puede no tener, bajo el punto de vista del propio usuario, un retorno suficiente.

\section{Es necesario proporcionar un acceso} único y alcanzar la interoperabilidad e integración de los servicios de préstamo digital con el resto de recursos ofertados por las bibliotecas públicas

Nuestra propuesta apunta, consecuentemente, hacia la necesidad de proporcionar un acceso único y de alcanzar la interoperabilidad e integración con el resto de recursos que también ofertan las bibliotecas públicas como los catálogos online. Tal y como establece la International Federation of Library Associations and Institutions (IFLA) en IFLA principles for library e-lending (IFLA, 2013), los libros electrónicos disponibles en las bibliotecas deben poder integrase en los opacs de éstas y ser interoperables a través de las plataformas, las aplicaciones y los dispositivos de estos centros y sus usuarios.

La respuesta a esta necesidad de integración de la consulta la dan las discovery tools. Ampliamente implantadas en las bibliotecas académicas españolas, las bibliotecas públicas todavía no han dado el salto a las soluciones que aportan los servicios de descubrimiento. Frente al $79,7 \%$ de las bibliote- 
cas universitarias que en marzo de 2014 contaba con uno de estos programas o estaba inmersa en un proceso de selección (Ávila-García; Ortiz-Repiso; Rodríguez-Mateos, 2015), en 2017 apenas encontramos experiencias en bibliotecas públicas. Argus en Cataluña y Casba en Andalucía constituyen un intento de integración parcial ya que no indexan los ebooks disponibles en eBiblio u otros recursos electrónicos.

La tendencia actual en las bibliotecas se orienta hacia la mejora de sus propios catálogos online con capas de descubrimiento o la sustitución de éstos por interfaces que incorporan funciones de servicios de descubrimiento como Ebsco Discovery Service, BiblioCore, Summon, VuFind o Encore que indexan ebooks y otros recursos electrónicos (Breeding, 2016). De este modo el opac se mantiene como vía preferente de consulta a todos los recursos sin que el usuario deba salir del mismo para encontrar la información.

Más allá del acceso único, las bibliotecas públicas norteamericanas han planteado la necesidad de que también el préstamo digital pueda realizarse dentro de sus propios catálogos online con el fin de que el usuario no se aleje de la esfera de éstas y evitar que navegue hacia otros entornos. Por esta razón, algunas plataformas han dado soluciones tecnológicas a esta demanda mediante APIs que posibilitan la integración de funciones de préstamo de ebooks en el propio opac de la biblioteca (Breeding, 2014). Encontramos ejemplos de esta integración en las alianzas que los fabricantes de plataformas de préstamo digital como OverDrive, $3 M$ o Baker\&Taylor mantienen con proveedores de SIGB y de programas de descubrimiento (SirsiDynix, Innovative Intefaces, Ebsco o BiblioCommons) y que se materializan en los catálogos de las bibliotecas públicas de Nueva York, Washington D.C. o Chicago.

Enumeramos, a continuación, aquellos elementos que consideramos clave para el acceso único y la integración de las plataformas de préstamo digital con el resto de recursos de la biblioteca:

- El lector debe poder prestarse un ebook, reservar un documento físico de una biblioteca o leer un artículo de una revista identificándose una única vez mediante un servicio de auntenticación centralizado.

- El proveedor del servicio de descubrimiento debe mantener acuerdos con el mayor número de editores y distribuidores de contenidos de la biblioteca e indexar éstos en profundidad proporcionado metadatos, resúmenes, descriptores y, en la medida de lo posible, texto completo (Deodato, 2015).

- Con el fin de garantizar una mayor independencia y variedad en la oferta de títulos, es aconsejable que el proveedor del servicio de descubrimiento y el proveedor de los contenidos sean distintos.

- El grado de integración de los recursos dependerá de los acuerdos alcanzados por el proveedor del servicio de descubrimiento con los productores de las plataformas de préstamo digital y del SIGB de la biblioteca. La integración de los programas de descubrimiento con este último no conlleva problemas técnicos (Ávila-García; Ortiz-Repiso; Rodríguez-Mateos, 2015). En el caso de los recursos electrónicos puede ser parcial (redireccionamiento a la aplicación de origen) o total (préstamo y consulta desde el propio opac de la biblioteca).

- Al igual que para la colección física, el usuario debe poder obtener información en tiempo real sobre las copias y la disponibilidad tanto de los recursos electrónicos como de la colección en papel.

- Cualquiera que sea la decisión de la biblioteca (conservar su propio opac mejorado o renunciar a éste por la interfaz que proporciona el programa de descubrimiento), es conveniente preservar el máximo número de funciones que ya presentan las plataformas de préstamo digital (información sobre disponibilidad, formatos y opciones de lectura, estrategias de marketing como carruseles y recomendaciones personalizadas, participación del usuario, apps, etc.)

La integración no debe implicar la renuncia a las funciones que ya presentan las plataformas de préstamo de libro electrónico

\section{Conclusiones}

El acceso único y la integración se perfilan como elementos que pueden contribuir a mejorar la visibilidad de los ebooks e incrementar los índices de préstamo digital en las bibliotecas públicas españolas. La incorporación a los catálogos online de servicios de descubrimiento y de las funciones implementadas por las plataformas de préstamo digital constituye una vía con la que dar respuesta a la atomización y mejorar la experiencia del usuario sin que éste deba abandonar el contexto que le ofrece la biblioteca a través del opac.

El sistema de indicadores expuesto es aplicable a la evaluación de la información, servicios y funciones que estos opacs mejorados proporcionan a los usuarios. Es necesaria, por tanto, una evaluación ulterior de las experiencias ya existentes en las bibliotecas públicas norteamericanas.

Así mismo, y con el fin de analizar otros factores que también pueden condicionar el éxito del servicio de préstamo digital en España, se requiere un estudio complementario que profundice en las percepciones y prácticas lectoras de los propios usuarios de nuestras bibliotecas públicas.

\section{Notas}

1. El informe eBiblio 2015 no incluye los datos de eLiburutegia en el País Vasco y del Catálogo gallego de GaliciaLe, así como los de otras plataformas implantadas en redes y bibliotecas que han decidido proporcionar este servicio de manera autónoma con otros proveedores. Baleares, Melilla y Cataluña se incorporaron a eBiblio a lo largo de 2015.

2. Al igual que en 2015, en los informes eBiblio 2016 y 2017 no se recogen los datos de eLiburutegia en el País Vasco, los del Catálogo gallego de GaliciaLe ni los de otras plataformas de bibliotecas municipales, biblioteca de diputaciones, etc.

3. Este tipo de directorios sigue un protocolo LDAP (Lightweight directory access protocol) y se usa sobre todo para almacenar y buscar información sobre personas. Cada objeto (usuario) es descrito por una entrada consistente en una 
colección de atributos (nombre, dirección, teléfono, etc.). Permite, además, una autenticación única para varios servicios (por ejemplo, en el caso de las bibliotecas públicas gallegas, los usuarios acceden a su sesión personal en la plataforma de préstamo de libro electrónico gallego y en el opac del SIGB autenticándose contra un único directorio).

\section{Referencias}

Alonso-Arévalo, Julio; Cordón-García, José-Antonio (2015). "Retos en torno al préstamo de libros digitales en bibliotecas". Anales de documentación, v. 18, n. 1.

https://doi.org/10.6018/analesdoc.18.1.207171

Ávila-García, Lorena; Ortiz-Repiso, Virginia; Rodríguez-Mateos, David (2015). "Herramientas de descubrimiento: ¿una ventanilla única?". Revista española de documentación científica, v. 38, n. 1.

https://doi.org/10.3989/redc.2015.1.1178

Bawden, David; Vilar, Polona (2006). “Digital libraries: to meet or manage user expectations". Aslib proceedings, v. 58, n. 4, pp. 346-354.

https://tefkos.comminfo.rutgers.edu/Courses/e530/Readings/ Bawden\%20dl\%20based\%20on\%20LIDA\%202006.pdf https://doi.org/10.1108/00012530610687713

Breeding, Marshall (2014). "Discovery product functionality". Library technology reports, v. 50, n. 1, pp. 5-32.

https://journals.ala.org/index.php/Itr/article/view/5779/7237

Breeding, Marshall (2016). "Library systems report 2016: Power plays". American libraries, v. 47, n 5, pp. 30-43. https://americanlibrariesmagazine.org/wp-content/ uploads/2016/05/0516-american-libraries.pdf

Chickering, F. William; Yang, Sharon Q. (2014). "Evaluation and comparison of discovery tools: An update". Information technology and libraries, v. 33, n. 2, pp. 5-30.

https://doi.org/10.6017/ital.v33i2.3471

Codina, Lluís (2008). "Sistema general de análisis y evaluación de sitios web. Parámetros e indicadores". Lluiscodina. com, 7 octubre.

https://www.lluiscodina.com/wp-content/uploads/2014/04/ indicadores_2008.pdf

Dempsey, Lorcan (2005). "The sound of words: Amazoogle and Googlezon". Lorcan Dempsey's weblog, 29 enero. http://orweblog.oclc.org/archives/000562.html

Deodato, Joseph (2015). "Evaluating web-scale discovery services: a step-by-step guide". Information technology and libraries, v. 34, n. 2, pp. 19-75.

https://doi.org/10.6017/ital.v34i2.5745

Hoffert, Barbara (2017). "Under the surface. Materials survey 2017". Library journal, 23 febrero.

http://reviews.libraryjournal.com/2017/02/collectiondevelopment/under-the-surface-materials-survey-2017

IFLA (2013). IFLA principles for library elending. International Federation of Library Associations and Institutions. https://www.ifla.org/elending/principles

Kelley, Michael (2012). "Integrating ebooks". Library journal, v. 137, n. 7, pp. 46-47.

http://www.ala.org/tools/librariestransform/integratingebooks

Martindale, Graham; Willett, Peter; Jones, Roger (2015). "Use and perceptions of e-books in Derbyshire libraries". Library review, v. 64, n. 1/2, pp. 2-20.

https://goo.gl/CL2oCJ

https://doi.org/10.1108/LR-04-2014-0030

MECD (2016a). eBiblio 2015. Ministerio de Educación, Cultura y Deporte. Subdirección General de Coordinación Bibliotecaria.

http://www.mecd.gob.es/mecd/dms/mecd/cultura-mecd/ areas-cultura/bibliotecas/eBiblio/eBiblio_2015_est.pdf

MECD (2016b). Bibliotecas públicas españolas en cifras 2015. Ministerio de Educación, Cultura y Deporte. Subdirección General de Coordinación Bibliotecaria.

http://www.mecd.gob.es/cultura-mecd/areas-cultura/ bibliotecas/novedades/Estadisticas-2015.html

MECD (2017a). eBiblio 2016. Ministerio de Educación, Cultura y Deporte. Subdirección General de Coordinación Bibliotecaria.

http://www.mecd.gob.es/mecd/dms/mecd/cultura-mecd/ areas-cultura/bibliotecas/eBiblio/eBiblio_2016_est.pdf

MECD (2017b). Panorámica de la edición española de libros 2016. Ministerio de Educación, Cultura y Deporte. Subdirección General del Libro, la Lectura y las Letras españolas. https://sede.educacion.gob.es/publiventa/d/21061C/19/0

MECD (2018). eBiblio 2017. Ministerio de Educación, Cultura y Deporte. Subdirección General de Coordinación Bibliotecaria.

http://www.mecd.gob.es/dam/jcr:18fd9845-7170-40c4a27d-7f881ccffb52/eBiblio_2017_est.pdf

Muñoz-Egido, Daniel; Hernández-Pérez, Tony (2016). "Evaluación de la usabilidad en catálogos centrados en el usuario: una propuesta basada en heurísticas". BiD: textos universitaris de biblioteconomia i documentació, n. 37 (diciembre).

http://bid.ub.edu/es/37/munoz.htm

Novotny, Eric (2004). "I don't think I click: A protocol analysis study of use of a library online catalog in the internet age". College \& research libraries, v. 65, n. 6, pp. 525-537. https://doi.org/10.5860/crl.65.6.525

W3C (2008). Web content accessibility guidelines 2.0. Web Accessibility Initiative; World Wide Web Consortium.

https://www.w3.org/TR/WCAG20/

Yang, Sharon Q.; Wagner, Kurt (2010) "Evaluating and comparing discovery tools: how close are we towards next generation catalog?". Library hi tech, v. 28, n. 4, pp. 690-709. https://goo.gl/KUbW9H

https://doi.org/10.1108/07378831011096312 